\section{Monoecious Mutant Reveals New Insights into Male and Female Inflorescence Development in the Chinese Bayberry (Morella rubra)}

\author{
Zehuang Zhang ${ }^{1,2}$, Qihua Lin ${ }^{1}$, and Qiuzhen Zhong \\ Fruit Research Institute, Fujian Academy of Agricultural Sciences, Fuzhou \\ 350013, People's Republic of China
}

Additional index words. monoecism, inflorescence, RNA-seq

\begin{abstract}
To identify the dynamic differences in transcriptome and gene expression in chinese bayberry flowers of different sex types, the female 'Fugong-1' (WT) and its monoecious mutant (MT) were used as experimental materials. Using Illumina HiSeq $^{\mathrm{TM}}$ 2500, flowers at the inflorescence germination, inflorescence elongation, and initial flowering stages were sequenced by RNA-seq technology and 6 libraries were obtained. After sequence assemble, 84,945 unigenes greater than 200 bp were found and the total length was $71.66 \mathrm{Mb}$. Transcriptomic expression analysis of the six libraries indicated that there was only 297 genes showed different expression at the inflorescence germination stage between MT and WT, the difference of which was the minimum. At the elongation stage and the initial flowering stage, such numbers increased to 787 and 2722 , respectively. Gene ontology (GO) functional enrichment analysis revealed that the enriched differentially expressed genes (DEGs) included those related with transcription in DNA-templated (GO: 0006355), pollen exine formation (GO: 0010584), plasma membrane (GO: 0005886), sequence-specific DNA binding transcription factor activity (GO: 0003700), and 2-alkenal reductase [NAD(P)] activity (GO: 0032440) GO categories, etc. Among these processes, pollen exine formation plays an important role in pollen cell wall synthesis and sporopollen participates in the biosynthesis of sporopollenin. High expression levels of these related genes were closely related to the MT's male flower traits during the initial flowering stage, which resulted in the male characteristics in MT flower. This study provides important foundation for further mining important genes and regulating factors controlling the sex differentiation of flowers in chinese bayberry.
\end{abstract}

The gender of higher plants has been demonstrated in monoecious and androgyne (Oryza sativa and triticum), monoecious but androgyne (Zea mays and Ricinus communis), and dioecism plants (poplar and Cannabis sativa). Most of these higher plants are monoecious, since dioecism accounts for only $5 \%$ of the flowering higher plants (Lionakis, 1985). The evolutionary process initiated with monoecism and androgyne, evolved to monoecism but androgyne, and subsequently resulted in dioecism. In addition, flowers also evolved from bisexual to unisexual presentation (Durand and Durand, 1984; Irish and Nelson, 1989; Meng et al., 1995).

The chinese bayberry (Morella rubra, former name Myrica rubra) is a subtropical evergreen tree native to China and other

Received for publication 3 Oct. 2016. Accepted for publication 20 Jan. 2017.

This work was supported by the Special Fund for Agro-scientific Research in the Public Interest (201203089), and the Basic Research Projects of Fujian Provincial Public Research Institute (2016R1013-1, 2014R1014-12).

${ }^{1}$ These authors contributed equally.

${ }^{2}$ Corresponding author. E-mail: 7901817@sina. com.
Asian countries (Chen et al., 2004; Jia et al., 2015; Xie et al., 2011). Chinese bayberry is planted as an important fruit crop in China, and mainly cultivated in Zhejiang, Fujian, and Jiangsu provinces (Zhang et al., 2009). In 2015 , the planting area for chinese bayberry consisted of $\approx 230,000 \mathrm{hm}^{2}$ in China. Chinese bayberry $(2 n=16)$ belongs to the Myricaceae family, is usually dioecious, and is wind pollinated (Erickson and Hamrick, 2003; González-Pérez et al., 2009; Stokes, 1997). In addition, only a few individuals are monoecious (Jiao et al., 2013), although this phenomenon is very rare. The male and female populations of chinese bayberry have similar genetic diversity in terms of the average number of alleles and level of heterozygosity, but were clearly separated by genetic structure analysis due to two markers associated with sex type (Jia et al., 2015). In our previous studies on the chinese bayberry, we found one monoecious mutant in 'Fugong-1'. This mutant was stable for a few years across continuous generations in different locations (Lin et al., 2013, 2016). The gender-decision system of plants is complex and various, and is affected by sex chromosomes, sex determination genes, gene balance, and the environment (Delph, 2003). SRAP (sequence-related amplified polymorphism) identification showed that 'Fugong-1' was as close as $95.7 \%$ to its monoecious mutant based on genetic distance (Lin et al., 2013). However, which resulted in this phenomenon was not clear.

In recent years, transcriptome sequencing technology using RNA-seq, which is based on next-generation sequencing, has been widely used in a range of research fields and can provide a quick and convenient method to establish the basic platform of plant molecular studies without the need for corresponding sequenced genome information as a reference (Biao et al., 2015; Brautigam et al., 2011; Dubey et al., 2011; Merianne et al., 2014; Parchman et al., 2010; Shi et al., 2011; Wang et al., 2010; Wu et al., 2010). Feng et al. (2012) obtained 41,239 unigenes from three different ripened fruits by using RNA-seq. The authors also developed a visual network of genetic dynamic changes within the chinese bayberry during fruit development and during the ripening stages using interactive pathways analyses. Yang et al. (2015) revealed key insights into stamen and pistil development in wheat (Triticum aestivum L.) by identifying 206 DEGs that were highly correlated with their development. These genes included WM27B, DL, YAB1, YABBY4, WM5, CER1, and WBLH1, which had been previously implicated in flower development.

In this study, we used 'Fugong-1' chinese bayberry and its monoecious mutant inflorescence in three different developing stages as our targets and sequenced their transcriptome using RNA-seq technology. By using inflorescence transcriptomes at different developing stages in these varieties, we aimed to identify the genes related to gender development. This study also provides important data for further studies on the sex types of the chinese bayberry.

\section{Materials and Methods}

All samples were collected from the chinese bayberry ('Fugong-1' and its monoecious mutant) trees growing in Longhai County, Fujian Province, China.

The sequencing libraries were defined as follows: Library 1 (WT1) inflorescence germination stage of WT (Inflorescence length was 2-3 mm, 23 Dec. 2014); Sequencing Library 2 (WT2): inflorescence elongation of WT (Inflorescence length was 5-7 mm, 16 Jan. 2015); Sequencing Library 3 (WT3): initial flowering stage of WT (10 Feb. 2015); Sequencing Library 4 (MT1): inflorescence germination stage of MT (Inflorescence length was 2-3 mm, 6 Dec. 2014); Sequencing Library 5 (MT2): inflorescence elongation of MT (Inflorescence length was 5-7 mm, 31 Dec. 2014); and Sequencing Library 6 (MT3): initial flowering stage of MT (28 Jan. 2015). We chose three samples of each variety and picked 100 inflorescences from each sample (300 samples in total). All samples were mixed well and quickly frozen in liquid 
nitrogen. Samples were then moved to storage below $-80{ }^{\circ} \mathrm{C}$.

RNA extraction and quality detection. RNA from six samples was extracted according to the instruction manual of the Plant RNA kit (Omega, R6827-02). The extracted RNA was treated with RNasefree DNase I (TaKaRa Biotech Co., Ltd., Dalian, China) to remove residual DNA. The purity, concentration, and integrity of the RNA samples were detected by NanoDrop, Qubit 2.0, and Agilent 2100, respectively, to ensure that qualified samples were used to carry out transcriptome sequencing (Shan et al., 2008).

Construction of cDNA library and illumina sequencing. High-quality RNA samples from chinese bayberry were sent to Biomarker Technologies Corporation (Beijing, China) for cDNA library construction and sequencing. Magnetic oligo (dT) beads were used to enrich the poly (A) mRNA tails of four independent RNA samples. The enriched mRNA was fragmented into small pieces, which were prepared as templates for cDNA synthesis. Double-stranded cDNA was synthesized using SuperScript II, buffer, dNTPs, RNaseH, and DNA polymerase I. The cDNA fragments were purified using the QiaQuick PCR extraction kit. The short cDNA fragments were then subjected to end repair, adapter ligation, and agarose gel electrophoresis filtration. Suitable fragments were excised for polymerase chain reaction amplification. The six constructed cDNA libraries of chinese bayberry were sequenced using the Illumina HiSeq ${ }^{\text {TM }} 2500$ platform, and the sequencing read length was PE125.

Data filtering and de novo assembly. Adaptor sequences, duplicated sequences, ambiguous reads ('N'), and low-quality reads were removed to obtain highquality clean reads. Transcriptomes from six chinese bayberry datasets were separately assembled de novo using the Trinity Program (http://trinityrnaseq.sourceforge. net/). The contigs were clustered using TGICL software (Pertea et al., 2003) to yield unigenes (without $\mathrm{N}$ ) and to acquire nonredundant (nr) unigenes by removing redundancies.

Functional annotation of the assembled unigenes. The unigene sequences of the six chinese bayberry samples were searched using BLASTX against the NCBI nr protein and nucleotide (Nt) databases (http://www. ncbi.nlm.nih.gov), the Swiss-Prot protein database (http://www.expasy.ch/sprot), the KEGG Ortholog (KO) database (http:// www.genome.jp/kegg/pathway.html), the GO database (http://www.geneontology. org/), the Protein family (Pram) database (http://en.wikipedia.org/wiki/Protein_family), and the Cluster of Orthologous Group of proteins (KOG/COG) database (http://www. ncbi.nlm.nih.gov/COG). Further functionalenrichment analysis of DEGs was carried out using topGO (Bioconductor package for $\mathrm{R}$ ) (http://www.bioconductor.org/packages/release/ bioc/html/topGO.html).
Differential gene expression analysis. The FPKM (fragments per kilobase of exon per million fragments mapped) method eliminated the influence of different gene lengths and sequencing levels on the calculation of gene expression. A differential gene expression analysis of two samples (WT1 vs. MT1, WT2 vs. MT2, WT3 vs. MT3, WT1 vs. WT2, WT2 vs. WT3, MT1 vs. MT2, and MT2 vs. MT3) was also performed. FDR $\leq 0.01$ and fold change $\geq 2$ were set as the thresholds for significance of the gene expression difference between the two samples.

Quantitative real-time reverse transcription $P C R$ analysis. The first-strand cDNA was synthesized from $1.0 \mu \mathrm{g}$ DNA-free RNA using the AMV first-strand cDNA Synthesis Kit (SK2445) according to the manufacturer's protocol. The quantitative PCR (qPCR) mixture (20 $\mu \mathrm{L}$ per volume) was comprised of $10 \mu \mathrm{L}$ SYBR $^{\circledR}$ SG Fast qPCR Master Mix [BBI, B639273, $0.4 \mu \mathrm{L}$ of each primer $(10 \mu \mathrm{M})]$ (Supplemental Data 1), $2 \mu \mathrm{L}$ of cDNA, and 7.2 $\mu \mathrm{L}$ of RNase-free water. The reactions were performed on a real-time PCR instrument (ABI StepOnePlus) as follows: $3 \mathrm{~min}$ at $95^{\circ} \mathrm{C}$ followed by 45 cycles of $95^{\circ} \mathrm{C}$ for $7 \mathrm{~s}, 57^{\circ} \mathrm{C}$ for $10 \mathrm{~s}$, and $72{ }^{\circ} \mathrm{C}$ for $15 \mathrm{~s}$. The reactions were completed with a melting curve analysis program. The specificity of qPCR primers was confirmed by melting curve and sequencing of qPCR products. The expression was calculated by $2^{-\triangle \mathrm{Ct}}$ and normalized to the actin gene (MrACT, GenBank accession no. GQ340770) (Niu et al., 2010), and least significant differences $(\mathrm{a}=0.05)$ were calculated for mean separations using the Data Processing System (version 3.01).

\section{Results and Discussion}

Morphological observation of inflorescence in 'Fugong-1' and its monoecious mutant. 'Fugong-1' is the female and produces female flowers (Fig. 1C); however, the MT grows both female and male flowers, which is different from the traditional chinese bayberry. We observed the external forms of the two flower development at the inflorescence germination, inflorescence elongation, and initial flowering stages. We found that the monoecious mutant was the same as 'Fugong-1' at the inflorescence germination stage (Fig. 1D) and at the inflorescence elongation stage (Fig. 1E). During the flowering period, there was an obvious difference between them, as the 'Fugong-1' grew female flowers (Fig. 1C) with typical female characteristics and the MT grew male and female mixed inflorescence (Fig. 1F). The female flowers grew at the top and the male flowers grew at the bottom of the inflorescence; some typical male inflorescence and female inflorescence were also found in branches.

Sequencing production statistics and de novo assembly Trinity. Six libraries were obtained from these two varieties during their three different phases (Supplemental Data 2). The total clean reads from the six libraries of each sample were between 19.5 and $21.4 \mathrm{M}$, and the total $\mathrm{Nt}$ count ranged from 4.92 to 5.39 Gb. Compared with the completed chinese bayberry genome of $304.4 \mathrm{Mb}$ (Qi et al., 2015), the count was as much as 16 times greater in depth. The results show that the rate of accordance with Q20 was more than $92 \%$ and the rate of accordance with Q30 was more than $87 \%$; the rate of $\mathrm{N}$ was 0 and the rate of GC was between $46 \%$ and $47 \%$. These data demonstrate that the sequencing data in this study are of good quality.

De novo assembly Trinity is a standard of assembly quality. We obtained 84,945 All-unigene using the Reads Trinity. After analyzing the length distribution of Allunigene (Supplemental Data 3 and 4), we found that All-unigenes were longer than $200 \mathrm{bp}$; those between 200 and $300 \mathrm{bp}$ had the highest rate (up to $31.57 \%$ ) and those longer than $1000 \mathrm{bp}$ had a rate of $23.09 \%$. The total length of 84,945 All-unigenes was $\approx 71.66 \mathrm{Mb}$. In addition, the average length was $943.586 \mathrm{bp}$ and N50 was $1554 \mathrm{bp}$, further indicating that the splicing result was accurate.

Functional annotation and categorization. All unigene sequences were annotated using BLASTX against the NCBI nr protein database, GO, Kyoto Encyclopedia of Genes and Genomes (Kanehisa and Goto, 2000), COG (Tatusov et al., 2001), Protein family (Pfam), and UniProtKB/(Swiss-Prot, TrEMBL) (O'Donovan et al., 2002) to annotate the assembly as comprehensively as possible. A total of 30,597 unigenes $(36.02 \%)$ were annotated with a significance threshold of E-value $\leq 1 \mathrm{E}^{-5}$. The remaining unigenes $(63.98 \%)$ could not be annotated with known genes (Supplemental Data 5), which was most likely due to the shortage of relevant genetic data and the presence of short sequences $(31.57 \%<300 \mathrm{bp})$.

E-value and species distribution were also analyzed by evaluating the matched unigenes $(29,536)$ from the returned BLASTX results of the nr protein database. The E-value distribution $(41.7 \%)$ of the mapped sequences showed strong homology ranging from $1 \mathrm{E}^{-50}$ to $1 \mathrm{E}^{-5}$. In addition, $58.3 \%$ had strong homology $\left(\mathrm{E}<1 \mathrm{E}^{-50}\right)$ to available plant sequences (Fig. 2A). The species distribution of the top hits that matched the sequences showed that Vitis vinifera $(11.41 \%)$ had the greatest number of matches to chinese bayberry, followed by Theobroma cacao (8.11\%), Prunus mume (7.27\%), Prunus persica (5.63\%), Jatropha curcas $(3.64 \%)$, Morus notabilis (3.64\%), Dothistroma septosporum (3.01\%), Citrus sinensis $(2.99 \%)$, R. communis (2.64\%), and Populus trichocarpa (2.62\%) (Fig. 2B).

GO classification. The expressed chinese bayberry genes were searched against the GO database to categorize standardized gene functions. Of the 29,536 unigenes previously annotated to the $\mathrm{nr}$ database, 17,330 were assigned to three main GO categories (biological process, cellular component, or molecular function) and 52 subcategories 


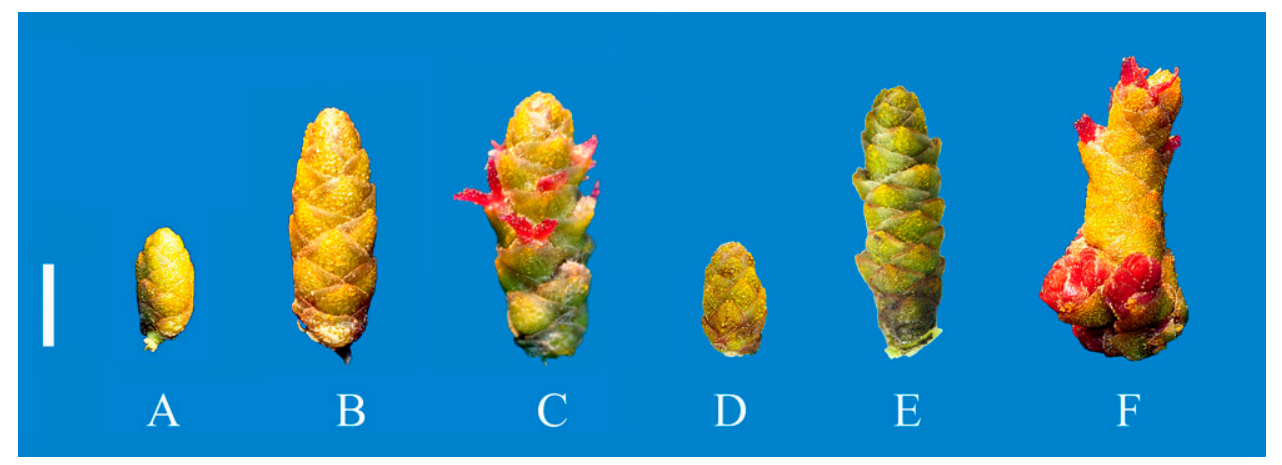

Fig. 1. Inflorescence for transcriptome sequencing of 'Fugong-1' and its monoecious mutant (MT). (A) inflorescence germination stage of WT; (B) inflorescence elongation stage of WT; (C) initial flowering stage of WT; (D) inflorescence germination stage of MT; (E) inflorescence elongation stage of MT; (F) initial flowering stage of MT; white bar $=2 \mathrm{~mm}$.
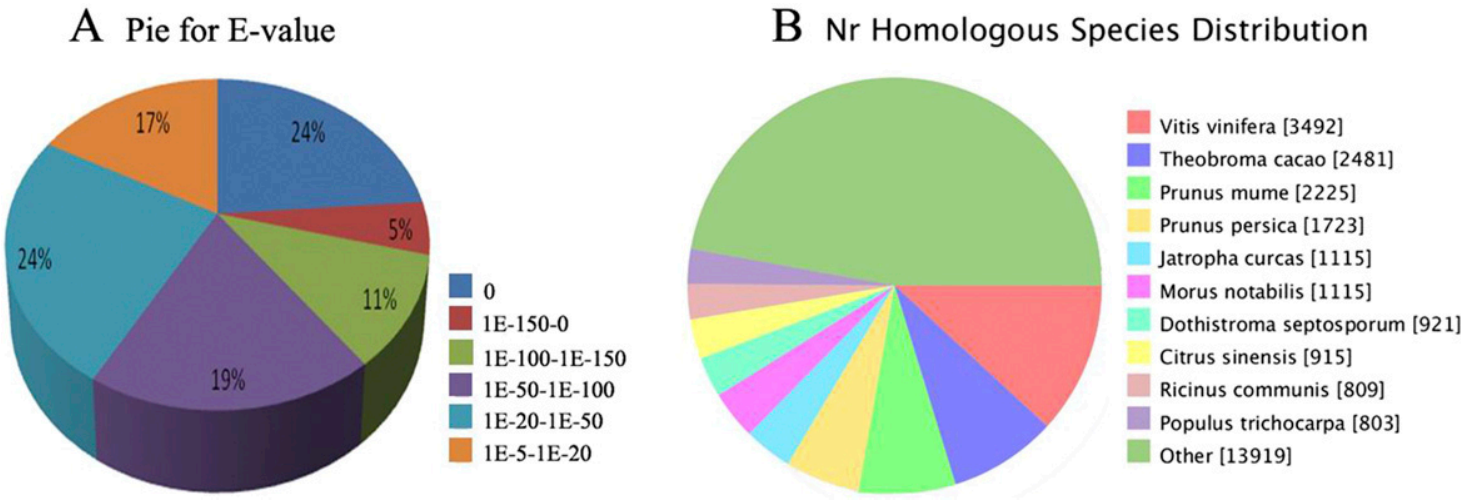

Fig. 2. Characteristics of homology search of chinese bayberry unigenes. (A) E-value istribution of BLASTX hits for each unigene; (B) number of UniGenes matching the 10 top species using BLASTx in the nr database.

using the Blast2GO and WEGO software (Fig. 3). A total of 4818 GO terms were collected, which were most frequently related to biological processes (2396), followed by molecular function (1937) and cellular components (485). The major subcategories among the biological processes were "metabolic process" (68.94\%; 11,948), "cellular process" (56.45\%; 9783), and "single-organism process" (47.48\%; 8228). "Catalytic activity" (56.20\%; 9740), "binding" $(51.03 \% ; 8843)$, and "transporter activity" $(7.23 \% ; 1253)$ were the dominant molecular functions. The most highly represented cellular components were "cell part" (39.78\%; 6894), followed by "cell” (39.6\%; 6863), "organelle" (29\%; 5027), "membrane" (22.60\%; 3916), "organelle part" (12.99\%; $2251)$, and "membrane part" (11.79\%; 2043).

Differentially expressed genes. Higher plants have a higher level of evolution and complex morphological and reproduction processes. They also have male and female gender, which is mainly expressed through flower production (Shou and Wang, 2000). Tandurdzic and Banks proposed that these differences in sex derived from the long-term evolution of monoecious plants (Tanurdzic and Banks, 2004) and that flowers resulted from unisexual evolution (Irish and Nelson, 1989; Meng et al., 1995). All stages of monoecious plant flower development require special genes; therefore, any structural gene mutation could cause the degradation or function loss of male and female organs, consequently leading to dioecism (Wang et al., 2004; Zhang et al., 2005). Therefore, this sex determination method may have formed during evolution to avoid inbred degradation and promote cross-pollination (Tanurdzic and Banks, 2004).

We identified the gene number of different expressions of 'Fugong-1' at three different phases of inflorescence development, the smallest of which was WT1 vs. WT2 at 689 and WT2 vs. WT3 at 715 (Table 1). The MT1 vs. MT2 was 707, which was similar to the number of different expressions at the inflorescence germination and inflorescence elongation stages; however, the number of MT2 vs. MT3 in monoecious mutant increased rapidly to 2527 . After calculating the number of different genes expressed in 'Fugong-1' and its monoecious mutant during the same budding phase (the number among WT1 vs. MT1, WT2 vs. MT2, and WT3 vs. MT3), we found that the number of different gene expressions during the MT1 and WT1 phases was the smallest (297). Previous studies have indicated that the number of MT and WT gene expressions has increased from 297 at the budding stage to 787 at the inflorescence elongation stage, reaching a maximum of 2722 at the initial flowering stage. These different genes concerning GO types include regulation of transcription, DNA-templated (GO: 0006355), pollen exine formation (GO: 0010584), sporopollenin biosynthetic process (GO: 0080110), plasma membrane (GO: 0005886), sequencespecific DNA binding transcription factor activity (GO: 0003700), and 2-alkenal reductase [NAD(P)] activity (GO: 0032440). These findings are likely explained by the fact that gene expression in MT related to male flower traits during the inflorescence germination stage had not started or was very limited. As the chinese bayberry gradually developed and flowered, most of the gene expression related to male flower development in MT started and became stronger. As a result, the number of differential genes at MT and WT increased.

GO-enrichment analysis of DEGs. After analyzing the differential gene expression of WT1 vs. WT2, WT1 vs. WT3, WT2 vs. WT3, MT1 vs. MT2, MT1 vs. MT3, MT2 vs. MT3, WT1 vs. MT1, WT2 vs. MT2, and WT3 vs. MT3, we found obvious enrichment of the pollen exine formation (GO: 0010584) in biological process at WT3 vs. MT3, MT1 vs. MT3, and MT2 vs. MT3 (Supplemental Data 6-8). These results indicate that differential genes expression between MT3 and MT1, MT2, and WT3 were related to the 


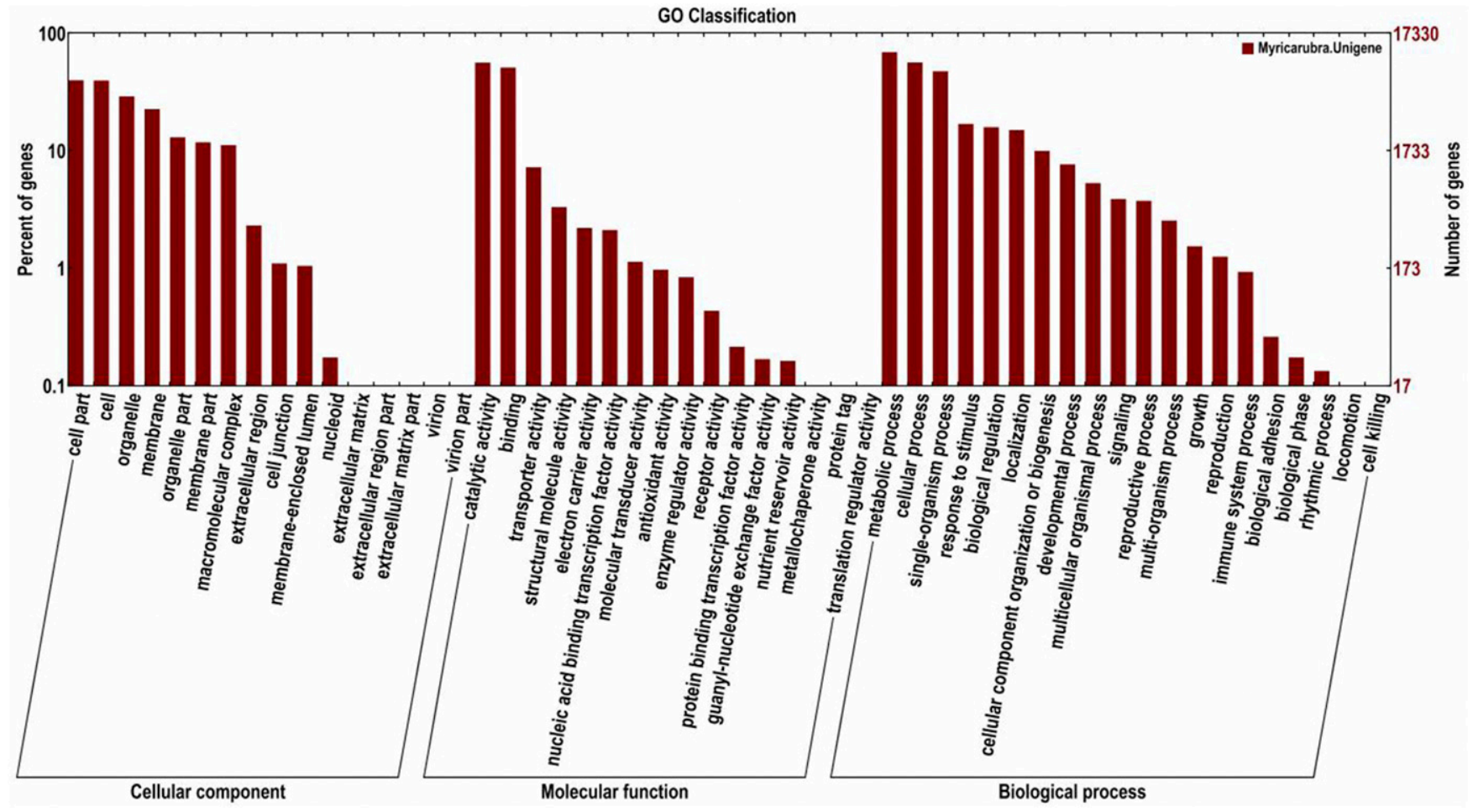

Fig. 3. Gene ontology (GO) classification of the chinese bayberry unigene library.

Table 1. Statistics of differential expression of genes among different samples.

\begin{tabular}{lccrrrrrr}
\hline DEG Set & Annotated & COG & \multicolumn{1}{c}{ GO } & KEGG & KOG & Pfam & Swiss-Prot & \multicolumn{1}{c}{ nr } \\
WT1_vs_WT2 & 689 & 379 & 345 & 173 & 343 & 580 & 495 & 629 \\
WT1_vs_WT3 & 1,297 & 446 & 813 & 253 & 588 & 1,013 & 1,053 & 1,296 \\
WT1_vs_MT1 & 297 & 106 & 175 & 61 & 143 & 221 & 217 & 296 \\
WT2_vs_WT3 & 715 & 393 & 365 & 165 & 338 & 608 & 501 & 652 \\
WT2_vs_MT2 & 787 & 405 & 414 & 184 & 375 & 652 & 557 & 729 \\
WT3_vs_MT3 & 2,722 & 927 & 1,554 & 457 & 1,348 & 2,160 & 2,089 & 2,713 \\
MT1_vs_MT2 & 707 & 232 & 436 & 112 & 293 & 557 & 562 & 706 \\
MT1_vs_MT3 & 2,874 & 997 & 1,620 & 466 & 1,436 & 2,222 & 2,162 & 2,863 \\
MT2_vs_MT3 & 2,527 & 863 & 1,431 & 407 & 1,272 & 1,958 & 1,905 & 2,513 \\
\hline
\end{tabular}

$\mathrm{DEG}=$ differentially expressed gene; $\mathrm{KOG} / \mathrm{COG}=$ Cluster of Orthologous Groups of proteins; KEGG = Kyoto Encyclopedia of Genes and Genomes; Pfam = Protein family; $\mathrm{nr}=$ nonredundant .

formation of the pollen wall. Expression was accelerated at the inflorescence later growth and kept the same pace with its morphological form. Due to much lower gene expression at the WT initial flowering stage (WT3) (Table 2), the morphology only showed female characteristics. We also found that all four unigenes (c30377.graph_c0, c51035. graph_c0, c42486.graph_c0, and c39830. graph_c0) of sporopollen in biosynthetic process (GO: 0080110) had higher expression levels during MT3 compared with WT3 (Table 3; Supplemental Data 9). The pollen exine formation (GO: 0010584) and sporopollenin biosynthetic process (GO: 0080110) are biological combination processes of pollen wall synthesis and sporopollenin, which mainly contribute to the formation of pollen. In the WT, there were only female flowers, and no male flowers were identified.

Although the 7 genes (c50948.graph c0, c47826.graph_c0, c43542.graph_c0, c35395. graph_c0, c22598.graph_c0, c19312.graph_c0, and c13541.graph_c0) in pollen exine formation (GO: 0010584) and 4 genes (c30377. graph_c0, c51035.graph_c0, c42486.graph_c0, and c39830.graph_c0) in sporopollenin biosynthetic process (GO: 0080110) at MT3 had higher expression levels than WT3, the expression levels of the 11 genes were tested during WT3. Low expression levels of some of the genes during WT1 and WT2 were also tested. These results indicate that the genes related to pollen formation already existed in the WT chinese bayberry genome, but the expression of these genes (which may include other genes related to the male flower formation) was inhibited. This inhibition caused the plant to exhibit female flower traits only. This evidence also indicated that the monoecious variation of the MT chinese bayberry was not because of a structural gene mutation, but was instead due to a regulatory reversal mutation. Further research and analyses are necessary to determine if the genes related to the male flower forma- tion also exist in other chinese bayberry sources, including the cultivated varieties, such as 'Dongkui', 'Biqi', 'Ruanshianhaibian', 'Raxiandao', and chinese bayberry subspecies, which include Morella nana, Morella adenophora, and Morella esculent.

Quantitative real-time reverse transcription $P C R$ analysis. Quantitative real-time reverse transcription PCR (qRT-PCR) was performed on 11 significantly upregulated unigenes in 'Fugong-1' monoecious mutants to validate the results of expression profiling obtained from RNA-seq. Among the unigenes selected from the GO significant enrichment category were pollen exine formation (GO: 0010584) and sporopollenin biosynthetic processes (GO: 0080110). We compared the results obtained from qRT-PCR with those generated from the RNA-seq analysis of the transcripts. The trends of expression were consistent for all transcripts in both analyses. The transcript levels in 'Fugong-1' monoecious mutant were 1.2-1053-fold higher than in 'Fugong-1' (Supplemental Data 10). We also chose four differential gene expressions from GO items 0010584 and 0080110 and analyzed their gene expressions in 'Fugong-1' monoecious mutant at different development phases (inflorescence germination stage, inflorescence elongation stage, and initial flowering stage). The qRT-PCR showed that these four genes in 'Fugong-1' monoecious mutant were all expressed during three developing phases (Fig. 4). The expressions of unigenes c19312.graph_c0 (polygalacturonase QRT3), c35395.graph_c0 (ABC transporter $\mathrm{G}$ family member 26), and c51035.graph_c0 
Table 2. Upregulated unigene expression in pollen exine formation.

\begin{tabular}{|c|c|c|c|c|c|c|c|}
\hline Unigene ID & WT3 & MT1 & MT2 & MT3 & $\log 2(\mathrm{MT} 3 / \mathrm{WT} 3)$ & $\log 2(\mathrm{MT} 3 / \mathrm{MT} 1)$ & $\log 2$ (MT3/MT2) \\
\hline c22598.graph_c0 & 2.0913 & 0.1980 & 0.1767 & 412.9836 & 7.7768 & 11.0794 & 11.2143 \\
\hline c35395.graph_c0 & 1.1790 & 0 & 0.0218 & 291.5716 & 8.0902 & 13.6029 & 12.7841 \\
\hline c47826.graph_c0 & 0.0814 & 0 & 0.0437 & 541.6133 & 12.1388 & 13.4944 & 12.6756 \\
\hline c50948.graph_c0 & 2.2385 & 1.5481 & 2.2972 & $2,016.979$ & 9.9597 & 10.5783 & 10.01 \\
\hline
\end{tabular}

Table 3. Upregulated unigene expression in sporopollenin biosynthetic processes.

\begin{tabular}{|c|c|c|c|c|c|c|c|}
\hline Unigene ID & WT3 & MT1 & MT2 & MT3 & $\log 2(\mathrm{MT} 3 / \mathrm{WT} 3)$ & $\log 2(\mathrm{MT} 3 / \mathrm{MT} 1)$ & $\log 2(\mathrm{MT} 3 / \mathrm{MT} 2)$ \\
\hline C30377.graph_c0 & 0.2284 & 0.0344 & 0.0701 & 558.6945 & 11.1789 & 13.0466 & 12.5141 \\
\hline C42486.graph_c0 & 0.7066 & 0.5092 & 0.7192 & 926.4602 & 10.4318 & 10.9631 & 10.4976 \\
\hline C51035.graph_c0 & 1.4323 & 0.0000 & 0.0942 & 809.1026 & 9.2759 & 14.5513 & 12.8224 \\
\hline
\end{tabular}

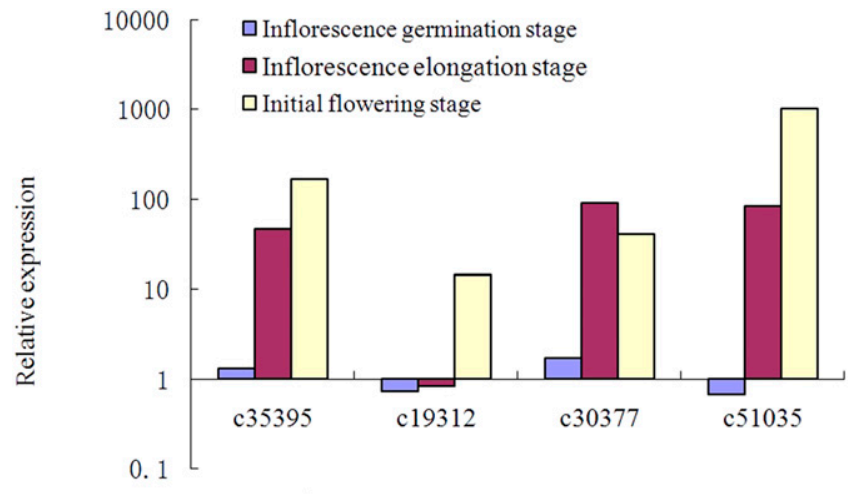

Different developmental stages

Fig. 4. Quantitative polymerase chain reaction detection results of four related genes in MT different stages.

(Cytochrome P450) gradually increased in the same direction with the flower development. Expressions of unigenes c30377.graph_c0 (tetraketide alpha-pyrone reductase 1) decreased during the initial flowering stage and rapidly increased at the full-bloom stage. The expressions of these four genes reached their highest levels at the full-bloom stage.

\section{Conclusion}

We sequenced transcriptomes of two sex type chinese bayberry and characterized gene expression profiles, examined differential expression profiles, and identified functional genes related to chinese bayberry male flower development. This work is significant for the development of genomic resources for chinese bayberry and provides important insights into the molecular mechanisms of chinese bayberry male flower development.

\section{Literature Cited}

Biao, L., H. Bing, H.Q. Yong, J.T. Zhao, H.C. Wang, and G.B. Hu. 2015. Transcriptomic analysis of Litchi chinensis pericarp during maturation with a focus on chlorophyll degradation and flavonoid biosynthesis. BMC Genomics 16:225.

Brautigam, A., T. Mullick, S. Schliesky, and P.M. Weber. 2011. Critical assessment of assembly strategies for non-model species mRNA-Seq data and application of next-generation sequencing to the comparison of $\mathrm{C}(3)$ and $\mathrm{C}(4)$ species. J. Expt. Bot. 9:3093-3102.

Chen, K.S., C.J. Xu, B. Zhang, and I.B. Ferguson. 2004. Red bayberry: Botany and horticulture. Hort. Rev. 30:83-114.

Delph, L.F. 2003. Sexual dimorphism in gender plasticity and its consequences for breeding system evolution. Evol. Dev. 1:34-39.

Dubey, A., A. Farmer, and J. Schlueter. 2011. Defining the transcriptome assembly and its use for genome dynamics and transcriptome profiling studies in Pigeonpea (Cajanus cajan L.). DNA Res. 3:153-164.

Durand, R. and B. Durand. 1984. Sexual differentiation in higher plants. Physiol. Plant. 60:267-274.

Erickson, D.L. and J.L. Hamrick. 2003. Genetic and clonal diversity for Myrica cerifera along a spatiotemporal island chronosequence. Heredity $90: 25-32$.

Feng, C., M. Chen, C.J. Xu, B. Lin, X.R. Yin, X. Li, A.C. Allan, I.B. Ferguson, and K.S. Chen. 2012. Transcriptomic analysis of Chinese bayberry (Myrica rubra) fruit development and ripening using RNA-Seq. BMC Genomics 13:19.

González-Pérez, M.A., P.A. Sosa, E. Rivero, E.A. González, and A. Naranjo. 2009. Molecular markers reveal no genetic differentiation between Myrica rivas-martinezii and M. faya (Myricaceae). Ann. Bot. (Lond.) 103(1):79-86

Irish, E.E. and T. Nelson. 1989. Sex determination in monoecious and dioecious plants. Plant Cell 1:737-744.
Jia, H.M., Y. Jiao, G.Y. Wang, Y.H. Li, H.J. Jia, H.X. Wu, C.Y. Chai, X. Dong, Y. Guo, L. Zhang, Q.K. Gao, W. Chen, L.J. Song, E. Weg, and Z.S. Gao. 2015. Genetic diversity of male and female Chinese bayberry (Myrica rubra) populations and identification of sexassociated markers. BMC Genomics 16:394.

Jiao, Y., G.Y. Wang, C.Y. Chai, H. Jia, and Z.S. Gao. 2013. Morphlogy of pollen grains from the plants with different type of sex by scanning electron microscope (SE) and the Viability of pollens in red bayberry. South Chin. Fruits 1:12-15.

Kanehisa, M. and S. Goto. 2000. KEGG: Kyoto Encyclopedia of Genes and Genomes. Nucleic Acids Res. 28:27-30.

Lin, Q.H., Q.Z. Zhong, and Z.H. Zhang. 2013. Genetic diversity analysis of 18 Chinese bayberry germplasm resources with SRAP. Redai Zuowu Xuebao 9:1667-1671.

Lin, Q.H., Q.Z. Zhong, and Z.H. Zhang. 2016. Research on fruit color of 'Fugong-1' and its early-maturing mutant Chinese bayberry ( $\mathrm{Myr}$ ica rubra) during development. South Chin. Fruits 1:107-109.

Lionakis, S.M. 1985. Genetics and physiology of sex determination in dioecious plants. Fruits 11:739-743.

Meng, J.L., D.F. Liu, and P. Luo. 1995. Plant reproductive genetics. Sci. Press, Beijing, China.

Merianne, A., J. Uwe, D. Myriam, S.V. Nocker, and M. Knoche. 2014. Transcriptional dynamics of the developing sweet cherry (Prunus avium L.) fruit: Sequencing, annotation and expression profiling of exocarp-associated genes. Hort. Res. 1:11.

Niu, S.S., C.J. Xu, W.S. Zhang, B. Zhang, X. Li, K.L. Wang, I.B. Ferguson, A.C. Allan, and K.S. Chen. 2010. Coordinated regulation of anthocyanin biosynthesis in Chinese bayberry (Myrica rubra) fruit by a R2R3 MYB transcription factor. Planta 4:887-899.

O’Donovan, C., M.J. Martin, A. Gattiker, E. Gasteiger, A. Bairoch, and R. Apweiler. 2002. High-quality protein knowledge resource: SWISS-PROT and TrEMBL. Brief. Bioinform. 3:275-284.

Parchman, T.L., K.S. Geist, J.A. Grahnen, C.W. Benkman, and C.A. Buerkle. 2010. Transcriptome sequencing in an ecologically important tree species: Assembly, annotation, and marker discovery. BMC Genomics 11:180.

Pertea, G., X.Q. Huang, F. Liang, V. Antonescu, R. Sultana, S. Karamycheva, Y. Lee, J. White, F. Cheung, B. Parvizi, J. Tsai, and J. Quackenbush. 2003. TIGR gene indices clustering tools (TGICL): A software system for fast clustering of large EST datasets. Bioinformatics 5:651-652 
Qi, X.J., H.Y. Ren, S.M. Liang, X.L. Zheng, and Y.C. Wu. 2015. Preliminary report on the results of whole genome sequencing of Chinese bayberry (Myrica rubra). J. Zhejiang Agr. Sci. 10:1564-1566.

Shan, L.L., X. Li, P. Wang, C. Cai, B. Zhang, C.D Sun, W.S. Zhang, C.J. Xu, I. Ferguson, and K.S. Chen. 2008. Characterization of cDNAs associated with lignification and their expression profiles in loquat fruit with different lignin accumulation. Planta 6:1243-1254.

Shi, C.Y., H. Yang, C.L. Wei, O. Yu, Z.Z. Zhang, C.J. Jiang, J. Sun, Y.Y. Li, Q. Chen, T. Xia, and X.C. Wan. 2011. Deep sequencing of the Camellia sinensis transcriptome revealed candidate genes for major metabolic pathways of tea-specific compounds. BMC Genomics 12:131.

Shou, S.Y. and Q.M. Wang. 2000. Progress of study on sex differentiation in higher plants. Chin. Bull. Bot. 6:528-535.

Stokes, J. 1997. Cytological studies in the Myricaceae-Contributions from the hull botanical laboratory 485. Bot. Gaz. 99:387-399.
Tanurdzic, M. and J.A. Banks. 2004. Sexdetermining mechanisms in land plants. Plant Cell 16:S61-S71.

Tatusov, R.L., D.A. Natale, I.V. Garkavtsev, T.A. Tatusova, U.T. Shankavaram, B.S. Rao, B. Kiryutin, M.Y. Galperin, N.D. Fedorova, and E.V. Koonin. 2001. The COG database: New developments in phylogenetic classification of proteins from complete genomes. Nucleic Acids Res. 1:22-28

Wang, H., L.V. Caruso, A.B. Downie, and S.E. Perry. 2004. The embryo MADS domain protein AGAMOUS-Like 15 directly regulates expression of a gene encoding an enzyme involved in gibberellin metabolism. Plant Cell 5:1206-1219.

Wang, X.W., J.B. Luan, J.M. Li, Y.Y. Bao, C.X Zhang, and S.S. Liu. 2010. De novo characterization of a whitefly transcriptome and analysis of its gene expression during development. BMC Genomics 11:400.

Wu, J., Y. Zhang, H. Zhang, H. Huang, K.M. Folta, and J. Lu. 2010. Whole genome wide expression profiles of Vitis amurensis grape responding to downy mildew by using Solexa sequencing technology. BMC Plant Biol. 10:234.

Xie, R.J., J. Zhou, G.Y. Wang, S.M. Zhang, L. Chen, and Z.S. Gao. 2011. Cultivar identification and genetic diversity of Chinese bayberry (Myrica rubra) accessions based on fluorescent SSR markers. Plant Mol. Biol. Rpt. 29:554-562.

Yang, Z.J., Z.S. Peng, S.H. Wei, M. Liao, Y. Yu, and Z. Jang. 2015. Pistillody mutant reveals key insights into stamen and pistil development in wheat (Triticum aestivum L.). BMC Genomics 16:211.

Zhang, S.M., Z.S. Gao, C.J. Xu, K.S. Chen, and G.Y. Wang. 2009. Genetic diversity of Chinese bayberry(Myrica rubra Sieb. et Zucc.) accessions revealed by amplified fragment length polymorphism. HortScience 2:487-491.

Zhang, X., B. Feng, Q. Zhang, D. Zhang, N. Altman, and H. Ma. 2005. Genome-wide expression profiling and identification of gene activities during early flower development in Arabidopsis. Plant Mol. Biol. 58:401-419. 